the cure is completed. Give a few smart doses of purgative medicine. Dr. Harvey adds the subjoined:-

"Cune For Encysted Tumouns, or Wens of the Head, or other parts of the body, without cutting them out."-First, make a longitudinal cut along the scalp. This is performed with little loss of blood. Next press out the contents of the cyst, and apply, freely, alcohol in the cavity, with a camel's hair brush. 'Then place in the cavity, also, from two to six grains of nitrate of silver, and bring the edges together with strappings, when inflammation takes place. Should it inflame too much, apply cold-water dressings, and give a few doses of active purgative medicine. This plan has ever been found to complete the cure in a few days.

Fistula in Axo (blind external) can often be cured without cutting, by injecting alcohol the whole length of the sinus, three or four times a-day, until it brings on inflammation; when that takes place, the cure is generally completed in a short time. In full habits, bleeding by the arm should be practised, if required, and the bowels opened pretty freely, before the alcohol is injected. Should the inflammation become too severe, it should be regulated by poultice or cold-water dressings, and low diet should strictly be attended to.

College Green, Bristol, Jan. 1st, 1845.

\section{CASE OF ACUTE FUNGOUS DISEASE OF THE THIGH AND LEG.}

By Charles Parson, Esq. Surgeon, Godalming.

Wr. $\mathrm{D}-$, of this town, an intelligent and delicate boy, nine years of age, born of healthy parents, had about two years ago, (in the winter of 1842,) an attack of acute rheumatism in the lower extremities, from which he suffered greatly for six weeks. After this he attended his school as usual, when one day, while at play, he received a kick in the leg from one of his schoolfellows. Little notice was taken of this at the time, but about the latter end of the spring, (1843,) the mother of the boy perceived in him an indisposition to move about, and that, in so doing, he rather dragged his right leg after him. In the Angust following, he complained of pain in his right thigh, which induced his friends to require my attendance. On examination, I discovered a small tumour, about the size of a pigeon's egg, attached to the bone inside of the thigh, about three inches above the knee-joint. Any increase in its size was at first imperceptible, for the patient was able, for some time, to walk to my surgery two or three times a week, impeded only by a slight lameness. But presently, a rapid enlargement of the tumour took place, attended with great pain, and ultimately confined the patient to his bed for many weeks. In the last week, the thigh measured two feet eight inches in circumference, and presented the appearance thus delineated:

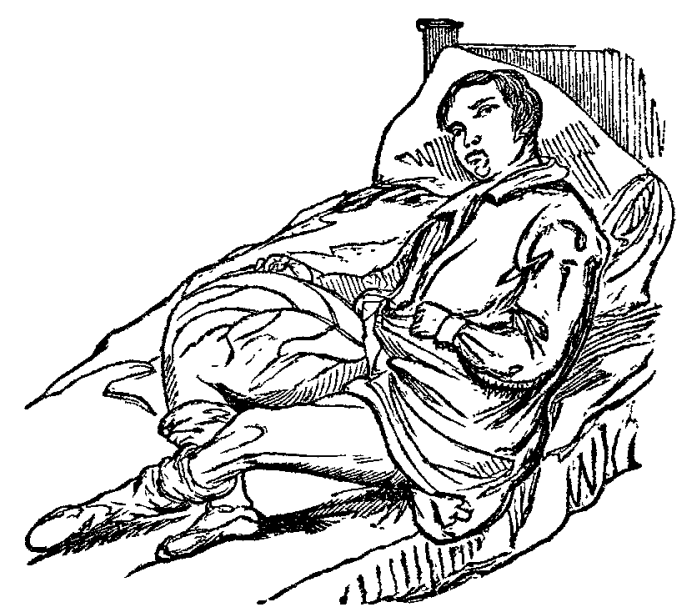

Two days before decease, foul sloughs broke out in the swelling, giving issue to a large quantity of bloody and brain-like discharge. Death terminated a scene of extreme suffering, Nov. 10th, 1843 .

EIn the early stage of the treatment, leeches, poultices, and anodyne fomentations were employed, as the tumour gave no indication of being other than an ordinary abscess. On its sudden increase, and having assumed the malignant character, tonics and opiates were had recourse to, and tincture of opium was taken to the amount of eighty drops in the twenty-four hours, without the slightest effect on the sensorium. A consultation of several surgeons was held, to consider the propriety of amputation, which was, how ever, deemed inadvisable.

In my absence, the post mortem examination, 'thirty-six hours after death, was conducted by my friend, Mr. Henry Taylor, of Guildford, who says, - "The body was much emaciated, but shewed no marks of decomposition, except in the affected limb. This was odematous in its whole length, and contrasted strongly with the attenuated state of the other limb. The tumour was seen to involve the whole of the thigh, the knee, and the upper third of the leg, though it had collapsed greatly, evidently, from the draining away of fluid by the ulcer's which had formed on the depending portion of its surface. The integument over it was green and wrinkled, and allowed the epiderwis to he peeled off readily. On making a longitudinal incision throughout the whole length of the tumour, the skin appeared as much softened as if it had been in maceration, and was of great thickness. The cellular membrane beneath was infiltrated with serum, and the superficial veins were turgid, and their coats much thickened. The fascia lata was greatly hypertrophied, and formed a kind of cyst for the contents of the tumour. On a puncture being made into it, a quantity of fotid gas escaped, allowing the tumour to collapse still further. The fascia was next slit up to an extent commensurate with the first incision, and a most confused mass presented itself. There were traces of emphysematous cellular membrane, mixed up with a dark-red pulpy matter, like disorganized lung, and the whole bathed in a bloody sanions fluid, like that which had escaped from the ulcers. It was impossible to recognise anything like muscular tissue or vessels. In the vicinity of the bone, the morbid matter was of much greater density, cutting like cartilage, with bony particles interspersed. Parts of the tumour, less broken down than the rest, presented the brain-like appearance of medullary disease. The thigh bone, on being dissected out, was found to have been fractured close above the condyles, where it had apparently been expanded into a cyst of some magnitude: its structure was much softened near this point, and readily admitted the entrance of a scalpel. The knee-joint had been extensively opened into, and its apparatus was completely disorganized. The museles of the calf were distinguishable below the tumour, but they were pale and softened, as by long maceration." I may add, that the ends of the diseased portion of the femur (which I still have by me) appear as if completely rotted asunder.

The above case resembles some related by Sir Charles Bell in his "Surgical Observations," 1816-17. Mr. Taylor remarks to me that it is difficult to solve whether the disease in this case had originated in the bone itself, or in the soft parts. In either case it had made much more progress in the latter.

Godalming, Dec. 1844.

\section{CENSUS OF CASES OF INFLUENZA.}

By Geonae Ross, Esq. Surgeon, London.

I HAVE used a popular term to designate an epidemic of the mucous surfaces, because it means nothing. A better reason cannot always be given for the use of a better word. One of my objects in collecting these cases was to shew the varying impressions of the epidemic influence on patients of different ages and idiosyncrasies ; for I believe that, by this means, a more corrtet knowledge can be attained of the value of atmospheric agencies on the health, than by any other. The infuenza is now rife, and as I see no difference in the present form of the malady from that which prevailed in the neighbourbood where I was practising in the winter of the year 1842 , I shall proceed to give an analysis of 110 cases, which at that time came under my treatment. Some of those cases will not be recognised as cases of influenza; but as I am satisfied that their exciting causes were the same, I shall range them together.

The cases are mainly divided into three distinct groups, according as the mucous membrane of the intestinal tube, the langs, or both, were implicated in the disease. They are as follow:-

Thirty-six cases of simple gastric fever.-Of these, twenty-one were adults, from 24 to 75 years of age. None of these had diarrhoea, unless under the operation of medicine; when, however, relaxation of the bowels was established by therapeutic means, it was occasionally severe. In a few cases there was obstinate constipation.

Eleven cases, from 6 to 15 years of age: no diarrhoea. One of these cases, a girl of eight years of age, suffered at the same time from chorea, which subsided as she recovered her health.

Four cases, from 3 to 5 years of age : of these, three laboured under diarrhoea.

Twenty-six cases of affection of the lungs.-Of these, three, between 25 and 53 years of age, were cases of simple catarrh; three, from 55 to 80 , cases of chronic bronchitis ; one of these, a man of 80 years, delirious. Fourteen were cases under three years of age ; one only terminated in pertussis.

Six were cases of phthisis : one, a girl under 5 years; the five 